\title{
Transcriptional Analysis of Stearoyl-Acyl Carrier Protein Desaturase Genes from Olive (Olea europaea) in Relation to the Oleic Acid Content of the Virgin Olive Oil
}

Farshid Parvini1 ${ }^{1,2,3 \dagger}$, M. Dolores Sicardo ${ }^{1}$, Mehdi Hosseini-Mazinani ${ }^{2}$, José M. Martínez-Rivas ${ }^{1, *}$ and M. Luisa Hernández ${ }^{1}$

${ }^{1}$ Department of Biochemistry and Molecular Biology of Plant Products, Instituto de la Grasa (IG-CSIC). Campus Universidad Pablo de Olavide, 41013 Sevilla, Spain ${ }^{2}$ National Institute of Genetic Engineering and Biotechnology, Tehran, Iran

${ }^{3}$ Faculty of Biological Science, Tarbiat Modares University, Tehran, Iran

\section{*Corresponding author:}

José M. Martínez-Rivas. E-mail: mrivas@cica.es. Phone: +34 954611550

${ }^{\dagger}$ Present address: Department of Cell and Molecular Biology, Faculty of Science, Semnan University, Semnan, Iran. 
Table S1. Gene accession numbers and sequences of primers pairs used for gene expression analysis by qRT-PCR in the present study

\begin{tabular}{|c|c|c|c|}
\hline Gene & $\begin{array}{l}\text { Accession } \\
\text { number }\end{array}$ & Sequence & $\begin{array}{l}\text { Amplicon } \\
\text { size (bp) }\end{array}$ \\
\hline OepSAD1 & KX196198 & $\begin{array}{l}\text { Forward: 5’- GAAGGTCAAGCAGGCATCAT-3’ } \\
\text { Reverse: 5’- CGCATTTCTTGACACAGACC-3’ }\end{array}$ & 159 \\
\hline OepSAD2 & KX196199 & $\begin{array}{l}\text { Forward: 5’- AGGAACGAGCACAATCAAGG-3’ } \\
\text { Reverse: 5’- TACAGACCAGATGGCGACAG-3’' }\end{array}$ & 162 \\
\hline OepSAD3 & KX196200 & $\begin{array}{l}\text { Forward: 5’- TGCCTCCAAGAATCAGACG-3' } \\
\text { Reverse: 5’- ATTGCAAGGGAACAAACTACG-3' }\end{array}$ & 157 \\
\hline OeUBQ2 & AF429430 & $\begin{array}{l}\text { Forward: 5’ - AATGAAGTCTGTCTCTCCTTTGG-3' } \\
\text { Reverse: 5'- AAGGGAAATCCCATCAACG-3' }\end{array}$ & 132 \\
\hline
\end{tabular}


Table S2. Accession number of the different stearoyl-ACP desaturases included in the

\section{phylogenetic tree analysis}

\begin{tabular}{|c|c|c|}
\hline Name & Accession number & Plant \\
\hline AtSAD1 & AY094014 & Arabidopsis thaliana \\
\hline AtSAD2 & AY056428 & Arabidopsis thaliana \\
\hline AtSAD3 & ВТ010447 & Arabidopsis thaliana \\
\hline AtSAD4 & ВT029294 & Arabidopsis thaliana \\
\hline AtSAD5 & NM_111130 & Arabidopsis thaliana \\
\hline AtSAD6 & AY128883 & Arabidopsis thaliana \\
\hline AtSAD7 & ВТ004625 & Arabidopsis thaliana \\
\hline AhSAD & AF172728 & Arachis hypogaea \\
\hline BrSAD & X60978 & Brassica rapa \\
\hline BnSAD & X63364 & Brassica napus \\
\hline CsaSAD & AFQ60943 & Camelina sativa \\
\hline CtSAD & M61109 & Carthamus tinctorius \\
\hline CISAD & EU131523 & Cinnamomun longipaniculatum \\
\hline CocoSAD & KF574390 & Cocos nucifera \\
\hline CsSAD & M59858 & Cucumis sativus \\
\hline EgSAD & XM_010929403 & Elaeis guineensis \\
\hline FaSAD & HQ⿻̄43517 & Fraxinus americana \\
\hline GbSAD & HQ694561 & Ginkgo biloba \\
\hline GmSAD1 & AY885234 & Glycine max \\
\hline GmSAD2 & AY885233 & Glycine $\max$ \\
\hline HaSAD16 & U91339 & Helianthus annuus \\
\hline HaSAD17 & U91340 & Helianthus annuus \\
\hline JcSAD & DQ084491 & Jatropha curcas \\
\hline LuSAD1 & JQ963139 & Linum usitatissimum \\
\hline LuSAD2 & JQ963149 & Linum usitatissimum \\
\hline MtSAD & ADE06393 & Macadamia tetraphylla \\
\hline OepSAD1 & KX196198 & Olea europaea \\
\hline OepSAD2 & KX196199 & Olea europaea \\
\hline OepSAD3 & KX196200 & Olea europaea \\
\hline PaSAD & AF116861 & Persea americana \\
\hline PpSAD & KF192317 & Pongamia pinnata \\
\hline RcSAD & M59857 & Ricinus comunis \\
\hline SiSAD & D42086 & Sesamun indicum \\
\hline SchSAD & M83199 & Simmondsia chinensis \\
\hline SaSAD & JX412962 & Solanum acaule \\
\hline ScaSAD & JX412963 & Solanum cardiophyllum \\
\hline ScoSAD & X78935 & Solanum commersonii \\
\hline StSAD & M91238 & Solanum tuberosum \\
\hline SoSAD & X62898 & Spinacea oleracea \\
\hline TcSAD1 & KP704662 & Theobroma cacao \\
\hline TcSAD2 & KP704663 & Theobroma cacao \\
\hline TcSAD3 & KP704661 & Theobroma cacao \\
\hline TcSAD4 & KP704664 & Theobroma cacao \\
\hline TcSAD5 & KP704665 & Theobroma cacao \\
\hline TcSAD6 & KP704666 & Theobroma cacao \\
\hline TcSAD7 & KP704667 & Theobroma cacao \\
\hline TcSAD8 & KP704668 & Theobroma cacao \\
\hline TaSAD1 & HQ589252 & Triticum aestivum \\
\hline TaSAD2 & HQ589253 & Triticum aestivum \\
\hline XsSAD & KM107837 & Xanthoceras sorbifolia \\
\hline
\end{tabular}

